TITLE:

\title{
Quenching of Intrinsic Luminescence by Coloration in KI and RbI( Dissertation_全文)
}

$\operatorname{AUTHOR}(S)$ :

Hayashi, Tetsusuke

CITATION:

Hayashi, Tetsusuke. Quenching of Intrinsic Luminescence by Coloration in KI and RbI. 京 都大学, 1978, 理学博士

\section{ISSUE DATE:}

1978-01-23

URL:

https://doi.org/10.14989/doctor.r3474

RIGHT: 
学位申請論文

京都大学教善部助手

不哲华 


\title{
to be published in J. Phys. Soc. Japan Vol. 43, No. 5, 1977.
}

\author{
Quenching of Intrinsic Luminescence by Coloration \\ in $\mathrm{KI}$ and $\mathrm{RbI}$ \\ - Tetsusuke HAYASHI \\ Physics Laboratory, School of General Education, \\ Kyoto University, Kyoto 606 \\ ( Recelved June 8, 1977 )
}

\section{Sýnopsis}

The luminescence intensity measured under uv-excitation at low temperatures in X-irradiated $\mathrm{KI}$ and $\mathrm{RbI}$ has been compared with that measured in uncolored crystals. Reduction in the luminescence efficiency caused by the coloration is due to nonradiative annihilation of free excitons or conduction electron= hole pairs through interaction with V-centers. Change in the degree of the luminescence quenching observed with the increase in the photon energy of excitation from the exciton band region toward the interband transition region can be ascribed to the difference between the relaxation probability of excitons and of electron-hole pairs to the self-trapped exciton state. The temperature dependence of the luminescence quenching suggests that a part of free excitons relax to the self-trapped exciton state through thermal activation process 


\section{\$1. Introduction}

Intrinsic luminescence of alkali halide crystals observed with high efficiency at low temperatures is known to be due to radiative decay of so-called self-trapped excitons (STE) 1 ) The STE is the state resulting from lattice relaxation of an exciton or from recombination of a free electron with a self= trapped hole $\left(\mathrm{V}_{\mathrm{k}}\right.$-center) As for the self-trapping process of excitons, the possibility that a potential barrier exists between the free exciton state and the STE state was pointed out theoretically by Toyozawa ${ }^{2}$ and Sum1 et al. 3) Experimentally, Fontana et al. 4) explained the temperature dependence of the luminescence efficiency of $\mathrm{NaI}$ excited in the exciton band in terms of an exciton state separated from the STE state by a potential barrier. The similar idea was also used by Nishimura ${ }^{5}$ ) in order to explain the intrinsic luminescence of $\mathrm{KI}$, and by Lushchik et al ${ }^{6}$ ) in order to interprete the mechanism of the host-sensitized luminescence in some impurity-doped alkali halldes Recently, Kuusmann et al 7) observed a weak emission in alkali iodide crystals which lies close to the fundamental absorption edge, and they assumed it to come from a metastable free exciton state which lies above the STE state. These experimental works provide interesting informations about the possibility of a stable free exciton state in alkali halide, but are rather hypothetical.

It has been reported by several authors-10) that coloration with frradiation of uv-light or ionizin radiation induces quenching of luminescence in some alkili halicie crystals. 
Especially, Goldstein ${ }^{8}$ ) observed this phenomenon in the Intrinsic luminescence of $\mathrm{KI}$ excited in the exciton absorption band at $77 \mathrm{~K}$, and concluded that nonradiative annihilation of the excited state occurs with a probability proportional to the concentration of F-centers produced by irradiation. A similar experiment was performed by the present author and his coworkers ${ }^{1}$ ) at various temperatures from $10 \mathrm{~K}$ to $100 \mathrm{~K}$, and it was suggested that temperature dependence of the luminescence quenching reveals that of the self-trapping probability of excitons. These experiments were, however, restricted to excitation in the exciton absorption band region.

The present paper is concerned with quenching of the intrinsic luminescence by coloration in KI and RbI. Ratio of the luminescence intensity in colored crystals to that in uncolored crystals is studied under excitation with the light of various energies in the fundamental absorption band. Dependence of the ratio on temperature as well as on the concentration of defects is investigated. It is shown that behavior of the luminescence quenching obtained under excitation in the interband transition region is different from that obtained under excitation in the first exciton absorption band. The results are analyzed on the assumption that the luminescence intensity is proportional to the creation probability of the STE state, and the lattice relaxation process of excitons or free electron= hole pairs is discussed.

\section{§2. Experimental Procedures}




\section{Single crystals of $\mathrm{KI}$ were obtained from the Harshaw}

Chemical Co., while single crystals of RbI were grown in our laboratory by the Kyropoulos technique. Crystals of about $1 \mathrm{~mm}$ thick were cleaved and mounted on a copper holder of a cryostat Temperature of specimen was monitored by a thermocouple at 1 ched to the copper holder By rotation of the sample holder the crystal was able to faced toward various windows of the cryostat for X-ray irradiation, for optical absorption measurements and for luminescence excitation.

Excitation of luminescence was made with uv-light from a Seya-Namioka type vacuum monochromator in conjunction with a deuterium lamp through a LiF window of the cryostat. The bandpass was kept below $8 \mathrm{~A}$. The luminescence was detected at a right angle to the exciting light with an HTV-R636 photomultiplier tube through a Nikon 6250 grating monochromator or through appropriate filters

Coloration of crystals was made with $\mathrm{X}$-rays from a tungsten target tube operated at $40 \mathrm{kV}$ and $20 \mathrm{~mA}$ through a beryllium window of the cryostat The coloration was always carried out near room temperature after the luminescence in the uncolored crystal had been measured at low temperatures. Before the crystal was colored, and after each step of coloration, transmittance of light at various wavelengths was measured at $78 \mathrm{~K}$ in order to estimate the density of produced defects. The coloration did not occur uniformly into the crystal, but it seemed to occur apparently deep enough compared with the penetration depth of the monochromatic uv-light used for luminescence excitation. 
The number of F-center was estimated approximately from the optical density of F-absorption band with use of Smakula's formula. The irradiation with uv-light during luminescence studies brought negligible effect on the defect concentration.

\section{§3. Experimental Results}

Under Irradiation with uv-light in the fundamental absorption region, KI luminesces with high efficiency below $\approx 100 \mathrm{~K}$. Two emission bands are mainly induced at $3.31 \mathrm{eV}$ and $4.10 \mathrm{eV}$ under excitation in the interband transition region, while an emission band at $3.05 \mathrm{eV}$ is induced especially below $\sim 30 \mathrm{~K}$ in addition to the $3.31 \mathrm{eV}$ emission under excitation in the first exciton absorption band region. ${ }^{12)}$ These emission bands have been confirmed to originate from excited states of a self-trapped exciton. 13,14) After the crystal was colored, reduction in the intensity of each of these emission bands was observed at whole temperature range below $\sim 100 \mathrm{~K}$. No appreciable change was observed in the peak position or in the shape of these bands. The total intensity of these emission bands is used hereafter as the intensity of the STE luminescence. Excitation spectra of the total luminescence measured at $5 \mathrm{~K}$ for an uncolored KI ( $\left.I_{0}\right)$ and for the colored one (I) are shown by solid curves in Fig. 1. Here the reflection loss at the crystal surface has not been corrected. The number of F-center in the colored crystal was estimated to be $1.72 \times 10^{16} / \mathrm{cm}^{3}$ Ratio of the luminescence intensity $\mathrm{I}_{\mathrm{I}} \mathrm{I}_{0}$ is also shown by the broken curve in Fig. 1. In the upper part of the figure the 
one-photon absorption spectrum at $10 \mathrm{~K}$ measured by Teegarden and Baldini ${ }^{15)}$ and the two-photon absorption spectrum at $4.2 \mathrm{~K}$ as well as the location of the band gap energy $E_{g}$ given by Hopfield and Worlock ${ }^{16}$ ) are shown for comparison. The value of the ratio $I / I_{0}$ increases with raising the photon energy of excitation from $\sim 6.2 \mathrm{eV}$ which is in the neighborhood of the threshold for two-photon absorption.

Intrinsic luminescence of $\mathrm{RbI}$ observed below $\sim 70 \mathrm{~K}$ consists of three emission bands at $3.95 \mathrm{eV}, 3.14 \mathrm{eV}$ and $2.30 \mathrm{eV}$ 17) The $3.95 \mathrm{eV}$ emission is absent in the case of excitation in the first exciton absorption region. Excitation spectra of the total luminescence measured at $5 \mathrm{~K}$ for an uncolored $\mathrm{RbI}\left(\mathrm{I}_{0}\right)$ and for the colored one (I) are shown by solid curves in Fig. 2 The broken curve shows the ratio of the intensity $I / I_{0}$. Here the number of F-center in the colored crystal was $1.18 \times 10^{16}$, $\mathrm{cm}^{3}$ One- and two-photon absorption spectra and the band gap energy 15,18) are also shown for comparison. The increase in the value of $I / I_{0}$ appears above $\sim 6.1 \mathrm{eV}$ which is in the neighborhood of the two-photon absorption edge, and it is also nearly coincident with the threshold for intrinsic photoconductivity measured by Huggett and Teegarden. ${ }^{19}$ ) It is clear in both KI and $\mathrm{RbI}$ that the luminescence is strongly quenched by coloration especially when the luminescence is excited around the first exciton absorption band.

It should be noted that, in the case of excitation above $\approx 6.2 \mathrm{eV}$ for $\mathrm{KI}$ or above $\sim 6.1 \mathrm{eV}$ for RbI, the luminescence intensity rises somewhat slowly to a stationary value when the 
exciting light is turned on first after coloration. The time period for reaching the stationary value becomes longer as the exciting light intensity decreases. After the luminescence intensity reaches once the saturated value, the response is instantaneous when the exciting light is turned off or turned on again. Such initial slow build-up of the luminescence intensity in the colored crystal does not appear under exc1tation in the exciton band region. It is suggested from observation of the similar effect in impurity-doped alkall halides 20,21$)$ that the slow build-up of the luminescence is due to trapping of free electrons and holes by some centers created with $\mathrm{X}$-ray irradiation which may be associated with traces of impurities In the present paper, the luminescence intensity of colored crystals $I$ is always given by the saturated value.

The total Iuminescence intensity in an uncolored $\mathrm{KI}$ is nearly constant belo $\left.\mathrm{W}_{\wedge} \sim 80 \mathrm{~K}, 12\right)$ while it depends strongly on temperature in colored crystals especially if the excitation is made in the first exciton absorption band region. Typical examples are shown in Fig. 3. The temperature dependence obtained under excitation with $6.01 \mathrm{eV}$ photons is shown in (a), and that obtained under excitation with $637 \mathrm{eV}$ photons is shown in (b) The number of F-center in the colored crystal was $3.64 \times 10^{16} / \mathrm{cm}^{3}$ in both (a) and (b) Figure 4 shows the temperature dependence of the intensity ratio $I_{/} I_{0}$ obtained from values in Fig. 3. Closed circles and open circles correspond to values obtained for $6.01 \mathrm{eV}$ excitation and for $6.37 \mathrm{eV}$ excitation, respectively The excitation at 601 evitypical of 
the excitation in the exciton band. It was confirmed that the temperature dependence of $I / I_{0}$ is quite similar to the result for $6.01 \mathrm{eV}$ excitation as far as the excitation is made in the high energy side of the first exciton absorption band, " while the temperature dependence becomes weak as the photon energy of excitation is raised toward the interband transition region beyond the first exciton band. The excitation at $6.37 \mathrm{eV}$ is typical of the interband excitation.

* The study of the luminescence quenching under excitation in the low energy side of the first exciton band is omitted in the present work. Because a strong absorption change is induced by coloration in this energy region which is called $\gamma$-band, ${ }^{22}$ ) and it brings unavoidable complications.

In the case of RbI the total luminescence intensity in an uncolored crystal is nearly constant below $\sim 60 \mathrm{~K}$, while the intensity in the colored one varies with temperature in a similar way to the case of KI. Temperature dependence of the intensity ratio $I / I_{0}$ obtained under excitation in the exciton band $(5.98$ $\mathrm{eV})$ and under excitation above the band gap energy $(6.26 \mathrm{eV})$ is shown by closed circles and open circles, respectively, in Fig. 5 . Here the number of F-center in the colored crystal is $1.85 \times 10^{16}$ $1 \mathrm{~cm}^{3}$ It appears in both $\mathrm{KI}$ and $\mathrm{RbI}$ that the strong temperature dependence of the luminescence quenching is characteristic of exciton excitation.

Quenching of luminescence by coloration depends also on the 
density of defects. Coloration with $\mathrm{X}$-rays at room temperature produces mainly F-center and V-center. 22 ) Since optical absorption measurements proved that $V$-centers are produced in numbers proportional to F-centers, the number of F-center is used here as a measure of the defect concentration. The quantities $\left[\left(I_{0} / I\right)-1\right]$ derived from values of $I / I_{0}$ obtained at various temperatures under excitation with $6.01 \mathrm{eV}$ photons are plotted as a function of F-center density in Fig. 6 . It is seen that values of $\left[\left(I_{0} / I\right)-1\right]$ depend linearly on F-center density, so that the ratio $I / I_{0}$ can be given by

$$
I / I_{0}=\frac{1}{1+\alpha \cdot n_{F}} \text {, }
$$

here $\mathrm{n}_{\mathrm{F}}$ is the F-center density and $\alpha$ is a constant. In Fig. 7 values of $\left[\left(I_{0} / I\right)-1\right]$ obtained at $5 \mathrm{~K}$ are plotted agalnst F-center density in various cases of different values of excitation energy $(6.01 \mathrm{eV}, 6.26 \mathrm{eV}, 6.37 \mathrm{eV}$ and $656 \mathrm{eV})$ The values obtained for $6.26 \mathrm{eV}, 6.37 \mathrm{eV}$ and $6.56 \mathrm{eV}$ excitation are somewhat apart from the linear relation in contrast to the case of 6.01 eV excitation as shown by dashed curves Similar results were obtained for $\mathrm{RbI}$. The relation (1) was confirmed to be applicable when the excitation was made in the exciton band region of $\mathrm{RbI}$ below $\sim 60 \mathrm{~K}$.

In addition to experiments on the luminescence intensity as shown above, decay time of the $3.31 \mathrm{eV}$ emission and of the $3.05 \mathrm{eV}$ emission in colored $\mathrm{KI}$ was measured at $5 \mathrm{~K}$ and $78 \mathrm{~K}$ with use of a pulsed light from a spark source. All of observed 
decay curves were single exponential in time range longer than $\sim 1$ usec. after pulsed excitation. No appreciable change was found to be induced by coloration in the decay time of each emission even when the intensity of each emission was reduced by coloration down to $1 / 10$ The decay time of the $3.31 \mathrm{eV}$ emission at $78 \mathrm{~K}$ was $\sim 1.8 \mu \mathrm{sec}$. irrespective of the photon energy of excitation between $5.8 \mathrm{eV}$ and $65 \mathrm{eV}$ At $5 \mathrm{~K}$ the decay time of the $3.05 \mathrm{eV}$ emission excited with $6.0 \mathrm{eV}$ photons and that of the $3.31 \mathrm{eV}$ emission excited with $6.5 \mathrm{eV}$ photons were $\sim 6.0 \mu \mathrm{sec}$. and $\sim 5.5 \mu$ sec., respectively. These values are equal to those measured in the uncolored crystal, and are nearly colncident with those reported by other authors. ${ }^{14,23)}$

54. Analysis and Discussion

Irradiation with $\mathrm{X}$-rays near room temperature produces not only F-centers but also V-centers. Goldstein ${ }^{8)}$ found that no luminescence quenching occurs in the case of electrolytically colored KI, and concluded that the defect which plays a role on the luminescence quenching is not an isolated F-center. The present author and his coworkers ${ }^{24}$ ) reported the luminescence quenching in $\mathrm{KI}$ induced by uv-coloration below $\sim 200 \mathrm{~K}$, and showed that the quenching phenomenon is concerned with the presence of vacancies or V-centers As vacancies are not stable at room temperature, it must be true in the present case that the presence of $V$-centers is responsible for the luminescence quenching.

Optical absorption band due to V-centers appears from $\sim 2.5$ 
$\mathrm{eV}$ to $\sim 5.0 \mathrm{eV}$ with no well-defined structure in both $\mathrm{KI}$ and $\mathrm{RbI}$. Most of intrinsic emission bands appear in the energy region of the V-absorption band of each crystal, so that one might consider that the observed reduction in the luminescence intensity is due to reabsorption of emitted photons by V-centers. However, if $1 t$ were true, the remarkable dependence of the luminescence intensity in colored crystals on the excitation energy or on temperature should not be expected. As the optical density of V-absorption band measured in the present experiment is very small (less than $\sim 0.05$ ), the reabsorption effect is considered to be negligible.

Decay time measurements performed for KI supports the Inference that the nonradiative annihilation of intrinsic excitation occurs not through relaxed excited states from which photons are emitted. If it were the case, the decay time of the emission should be reduced by coloration. As mentioned in the preceding section, behaviors of the luminescence quenching by coloration depend on whether the excitation is made in the exciton band or in the interband transition region. This also suggests that the annihilation process occurs not through the STE state but in an early stage before the self-trapping.

\subsection{Excitation in the exciton band}

Let us first discuss the luminescence quenching observed under excitation in the exciton absorption region with use of a simple exciton decay model. Namely, let us assume that a part of excitons are annihilated nonradiatively with a proba- 
billty $1 / \tau_{D}$ due to the interaction with V-centers; let $1 / \tau_{s t}$ be the probability of exciton relaxation to STE states; and let $1 / \tau_{n r}$ be that of other nonradiative decays. If we assume that the total intensity of the STE luminescence is proportional to $1 / \tau_{\text {st }}$, the intensity I can be expressed by

$$
I \propto \frac{1 / \tau_{s t}}{I / \tau_{s t}+1 / \tau_{D}+1 / \tau_{n r}}
$$

In addition, assuming that $1 / \tau_{D}$ is proportional to the concentration of V-centers, and so that to the concentration of $F=$ centers $\left(1 / \tau_{D}=c \cdot n_{F}\right)$, and that $\tau_{n r}>>\tau_{s t}$, we obtain the relation

$$
I / I_{0}=\frac{1}{I+c \cdot \tau_{s t} \cdot n_{F}}
$$

This is the similar relation as that derived by Goldstein ${ }^{8}$ in order to explain his experimental results obtained at $77 \mathrm{~K}$. The relation (3) coincides with the experimental relation (1) if we put $c \cdot \tau_{\text {st }}$ to $\alpha$.

Temperature dependence of the value of $c \cdot \tau$ st can be estimated from that of measured $I / I_{0}$ according to the relation (3) The obtained values are plotted as a function of $1 / T$ in Fig. 8 (a) and (b) The temperature dependence of $c \tau_{\text {st }}$ for each crystal can be approximated by the relation

$$
c \cdot \tau_{s t}=[A+B \cdot \exp (-\Delta E / k T)]^{-1}
$$


Solld curves in FIg. 8 (a) and (b) represent this relation, where constants $A, B$ and $\Delta E$ are chosen so as to fit the experimental points of each crystal. Values of energy $\Delta E$ chosen here are $18.0 \pm 0.5 \mathrm{meV}$, and $12.2 \pm 0.5 \mathrm{meV}$ for $\mathrm{KI}$ and for $\mathrm{RbI}$, respectively.

Assuming that the probability $1 / \tau_{D}\left(=c \cdot n_{F}\right)$ is independent of temperature, one can attribute the temperature dependence of $c \cdot \tau_{s t}$ to that of $\tau_{s t}$, and so that the self-trapping probability $1 / \tau_{\text {st }}$ can be represented from the relation (4) as the form

$$
1 / \tau_{s t}=1 / \tau_{s t}{ }^{\circ}+\nu \exp (-\Delta E / k T)
$$

This relation implies that a metastable free exciton state exists above STE states separated by a potential barrier $\Delta E$. Relaxation of excitons to STE states occurs on the one hand with a probability $1 / \tau_{s t}{ }^{\circ}$ which is independent of temperature, and on the other hand occurs thermally over the potential barrier. This conclusion is consistent with hypotheses given by other authors ${ }^{2-7}$ ) mentioned in $\$ 1$. It can be tentatively said that the temperature dependence of the luminescence quenching is governed by that of self-trapping probability of excitons. A part of excitons are trapped and annihilated near V-center sites efficiently at lower temperatures because they stay at the metastable state. This leads to the strong reduction in the STE luminescence. On the other hand, the increase in the self-trapping probability with the rise of temperature gives rise to the recovery of the luminescence intensity.

The above conclusion, which is based on the assumption 
that the probability $1 / \tau_{D}\left(=c \cdot n_{F}\right)$ is independent of temperature, is well supported by a previous paper on the edge emission in $\mathrm{KI}$ and $\mathrm{RbI}{ }^{25)}$ It was found that the temperature dependence of the intensity of the edge emission is quite similar to that of c. $\tau_{\text {st }}$ shown in Fig. 8. The efficiency of the edge emission was approximately given by $\tau_{s t} / \tau_{r}$, where $\tau_{r}$ is the radiative lifetime of a free exciton. The good agreement between the temperature dependence of $\tau_{s t} / \tau_{r}$ and of $c \cdot \tau_{s t}$ suggests that the temperature dependence of both quantities comes from that of $\tau_{\text {st }}$ as given by the relation (5).

\subsection{Excitation in the interband transition region}

As the photon energy of excitation is raised toward the interband transition region, the intensity ratio $I / I_{0}$ measured at $5 \mathrm{~K}$ increases as shown in Figs 1 and 2 as well as the temperature dependence of $I / I_{0}$ becomes weak as shown in Figs. 4 and 5 . Let us assume that pairs of conduction electrons and holes created with optical excitation relax to STE states through the following two processes. (1) The holes are localized to form self-trapped holes ( $V_{k}$-centers) and then recombination of electrons with self-trapped holes produces STE states. (2) A part of electron-hole pairs immediately create the lowest free excitons which is followed by the relaxation to STE states. The luminescence intensity $I_{0}$ is then given by

$$
I_{0}=I_{0}^{r e}+I_{0}^{e x}
$$

Here $I_{0}^{r e}$ is the intensity of the luminescence induced through 
the former process mentioned above, and $I_{0}{ }^{e x}$ is that induced through the latter process. The luminescence intensity I in the colored crystal is given by

$$
I=I^{r e}+I^{e x} \text {. }
$$

From the two equations we obtain

$$
I / I_{0}=\gamma \cdot\left(I^{e x} / I_{0}^{e x}\right)+(I-\gamma)\left(I^{r e} / I_{0}^{r e}\right)
$$

where

$$
\gamma=I_{0}^{e x} / I_{0}
$$

which corresponds to the production rate of the lowest free exciton.

The temperature dependence of $I / I_{0}$ obtained under excitation in the interband transition region can be explained in terms of the relation (6) on the assumption that the fraction $\gamma$ is independent of temperature and that the value of $I^{e x} / I_{0} e^{e x}$ depends on temperature in the same way as the intensity ratio $I / I_{0}$ obtained in the case of exciton excitation. In the case of $\mathrm{KI}$ the solid curve in Fig. 4 along experimental points for $637 \mathrm{eV}$ excitation is the calculated curve with use of the relation (6) Here values of $I / I_{0}$ obtained for $6.01 \mathrm{eV}$ excitation have been used as the values of $I^{e x} / I_{0}$ ex The ratio $I^{r e} / I_{0}$ re has been tentatively assumed to be independent of temperature. The value of $\gamma$ and that of $I^{r e} / I_{0}^{r e}$ chosen so as to fit the experimental points are 0.21 and 0.23 , respectively, in this case. The similar analysis was able to perform in the 
case of RbI. The solid curve along experimental points for $6.26 \mathrm{eV}$ excitation in Fig. 5 is the calculated curve from the relation (6). The value of $\gamma$ estimated is 0.15 .

Several data of the temperature dependence of $I / I_{0}$ were obtained in KI under various excitation near and above the band gap energy These results were all possible to be analyzed in the same way as above with use of the relation (6) based on the assumption that the ratio $I^{r e} / I_{0}{ }^{r e}$ is independent of temperature. Values of $\gamma$ chosen in these analyses are presented in Table I for several cases of different excitation energy. It is likely that the production rate of the lowest free exciton is less than $1 / 10$ of the total excitation when the excitation is made far above the band gap energy. Nouailhat et al. 26) reported that the quenching of luminescence by coloration is nearly independent of temperature in KI under excitation with electron beam. This seems consistent with the present result The dependence of the fraction $\gamma$ on excitation energy obtained here is consistent with the observation ${ }^{27}$ ) that the efficiency of the edge Iuminescence stimulated with uv-light decreases sharply with raising the photon energy of excitation from the exciton band region toward the interband transition region.

The value of $\left[\left(I_{0} / I\right)-1\right]$ obtained under excitation in the interband transition region appears not to be linear against F-center concentration as shown in Fig. 7 Such a fact also can be expected from the relation (6) It is clear that the ratio $I / I_{0}$ does not coincide with the relation (I) if the concentration dependence of $\mathrm{I}^{\mathrm{re}} / \mathrm{I}_{0}^{\mathrm{re}}$ is different irom that of 
$I^{e x} / I_{0}^{e x}$

It is concluded that pairs of cunduction electrons and holes created initially with excitation in the interband transition region relax to STE states through the two processes mentioned above A large part of them recombine to form the STE directly without passing through the lowest (metastable) free exciton state. The thermal activation process is not involved in this process, and the probability of the recombination must be independent of temperature in contrast to the case of exciton excitation. Before the relaxation to form the STE through either of the two processes, a part of excitons or of pairs of conduction electrons and holes are annihilated nonradiatively due to the interaction with V-centers, which leads to the quenching of luminescence. Differences in the behavior of the luminescence quenching observed with changing the photon energy of excitation from the first exciton absorption band into the interband transition region can be attributed to the change in the production rate of the lowest free exciton. It should be noted that there exsist absorption bands due to higher states of excitons in the interband transition region. However, no appreciable increase appeared in the production rate of the lowest free exciton $\gamma$ under excitation in these bands The creation of higher states of excitons is not able to be distinguished from the creation of free electron-hole pairs. 


\section{Acknowledgements}

The author wishes to express his sincere appreclation to Professor S.Koshino for his invaluable advice and encouragement throughout the course of this investigation. He would like to thank Miss $T$. Ohata for her continual help in experiments. 


\section{References}

1) M.N.Kabler: Point Defects in Solids, ed. J.H.Crawford,Jr. and L.M.Slifkin (Plenum Press, New York-London, 1972) Vol.1, p. 327 .

2) Y.Toyozawa: Progr. theor. Phys. 26 (1961) 29.

3) A.Sumi and Y.Toyozawa: J. Phys. Soc Japan 35 (1973) 137

4) M.P.Fontana, H.Blume and W.J.Van Sciver: Phys. Status Solidi $\underline{29}$ (1968) 159 .

5) H.Nishimura: J. Phys. Soc. Japan $\underline{38}$ (1975) 450.

6) Ch.Lushchik, G.Liidja, N.Lushchik, E.Vassil'chenko, K.Kalder, R.Kink and T.Soovik: Luminescence of Crystals, Molecules and Solutions, ed. F.Williams (Plenum Press, New York-London, 1973) p. 162.

7) I.L.Kuusmann, P.Kh.Liblik and Ch.B.Lushchik: JETP Letters 21 (1975) 72; I.L.Kuusmann, P.Kh.Liblik, G.G.Liid'ya, N.E.Lushchik, Ch.B.Lushchik and T.A.Soovik: Soviet Physics-Solid State 17 (1976) 2312 .

8) F T.Goldstein: Phys. Status Solidi 20 (1967) 379.

9) M.Ikeya and J.H.Crawford,Jr.: Phys. Letters 45A (1973) 213.

10) G.Guillot, A.Nouailhat, E.Mercier and P.Pinard: J. Luminescence $\underline{12 / 13}(1976) \quad 327$

11) T.Hayashi, T.Ohata and S.Koshino: Solid State Commun. 17 (1975) 945.

12) M.Ikezawa and T Kojima: J Phys. Soc. Japan 27 (1969) 1551

13) M.N.Kabler and D.A.Patterson: Phys. Rev. Letters 19 (1967) 652

14) T Yoshinari and M.Hirai: J Phys. Soc Japan 39 (1975) 1498

15) K.Teegarden and G.Baldini: Phys. Rev 155 (1967) 896. 
16) J.J.Hopfield and J.M.Worlock: Phys. Rev. 137 (1965) A1455.

17) J.Ramamuruti and K.Teegarden: Phys. Rev. 145 (1966) 698.

18) D.Fröhlich and B.Staginnus: Phys. Rev Letters 19 (1967) 496.

19) G.R.Huggett and K.Teegarden: Phys. Rev. 141 (1966) 797.

20) R.G.Kaufman and W.B.Hadley: J. chem. Phys. 44 (1966) 1311.

21) M.Itoh, T Tomi-e, K.Nakamura and Y.Nakai: J. Phys. Soc. Japan 41 (1976) 2052

22) J.D.Konitzer and H.N.Hersh: J. Phys Chem. Solids 27 (1966) 771.

23) J.U.Fischbach, D.Fröhlich and M.N.Kabler: J. Luminescence $\underline{6}$ (1973) 29 .

24) T.Hayashi, T.Ohata and S.Koshino: Solid State Commun. 18 (1976) 807 .

25). T.Hayashi, T.Ohata and S.Koshino: J Phys. Soc. Japan 42 (1977) 1647.

26) A.Nouailhat, G.Guillot and E.Mercier: Solid State Commun $\underline{21}(1977) 725$

27) T.Hayashi, T.Ohata and S.Koshino: J. Phys Soc Japan 43 (1977) 347 
Figure Captions

Fig. I Excitation spectra of the total luminescence measured at $5 \mathrm{~K}$ in an uncolored $\mathrm{KI}\left(I_{0}\right)$ and in the colored one (I) Broken curve shows the spectrum of the intensity ratio $I / I_{0}$ The F-center concentration in the colored crystal is $172 \times 10^{16} / \mathrm{cm}^{3}$ Arrows indicate the bandpass of the exciting monochromator. One- and two $=$ photon absorption spectra and the location of the band gap energy $E_{g}$ are shown for comparison in the upper part.

Fig. 2. Excitation spectra of the total luminescence measured at $5 \mathrm{~K}$ in an uncolored RbI $\left(I_{0}\right)$ and in the colored one (I) Broken curve shows the spectrum of the intensity ratio $I_{/} I_{0}$ The F-center concentration in the colored crystal is $118 \times 10^{16} / \mathrm{cm}^{3}$. Arrows indicate the bandpass of the exciting monochromator. One- and two= photon absorption spectra and the location of the band gap energy $\mathrm{E}_{\mathrm{g}}$ are shown for comparison in the upper part.

Fig. 3. Temperature dependence of the total luminescence intensity in an uncolored $K I\left(I_{0}\right)$ and in the colored one (I) measured under excitation with $6.01 \mathrm{eV}$ photons (a) and with $637 \mathrm{eV}$ photons (b) The F-center concentration in the colored crystal is $3.64 \times 10^{16} / \mathrm{cm}^{3}$ for both (a) and (b) 
Fig. 4. Temperature dependence of the intensity ratio $I / I_{0}$ obtained from values in $\mathrm{FIg}$. 3 for $6.01 \mathrm{eV}$ excltation (closed circles) and for $6.37 \mathrm{eV}$ excitation (open circles). The solid curve along experimental points for $6.37 \mathrm{eV}$ excitation is the calculated one in the text.

Fig. 5. Temperature dependence of the ratio of the luminescence intensity in a colored $\mathrm{RbI}$ to that in the uncolored one $\left(I / I_{0}\right)$ obtained under excitation with $5.98 \mathrm{eV}$ photons (closed circles) and with $6.26 \mathrm{eV}$ photons (open circles). The F-center concentration in the colored crystal is $1.85 \times 10^{16} / \mathrm{cm}^{3}$ in both cases. The solid curve along experimental points for $6.26 \mathrm{eV}$ excitation is the calculated one in the text

Fig. 6. Plot of quantities $\left[\left(I_{0} / I\right)-1\right]$ obtained in $K I$ as a function of the F-center concentration. The luminescence intensity, $I_{0}$ and $I$, was measured under $6.01 \mathrm{eV}$ excitation at various temperatures given in the figure.

Fig. 7 Plot of quantities $\left[\left(I_{0} / I\right)-1\right]$ obtained in $\mathrm{KI}$ at $5 \mathrm{~K}$ as a function of the F-center concentration. Photon energies of the luminescence excitation are given in the figure

Fig. 8 The value of $c \cdot \tau_{\text {st }}$ plotted as a function of $1 / T$ obtained for KI (a) and for RbI (b) The solid curve in each figure represents the calculated curve in the text. 
Table I. The production rate of the lowest free exciton $\gamma$ for various excitation energies in $K I$.

\begin{tabular}{c|c|c|c|c}
\hline $\begin{array}{l}\text { photon energy } \\
\text { of excitation }\end{array}$ & $6.26 \mathrm{eV}$ & $6.37 \mathrm{eV}$ & $656 \mathrm{eV}$ & $7.00 \mathrm{eV}$ \\
\hline$r$ & .0 .46 & 0.21 & 0.13 & 0.08 \\
\hline
\end{tabular}




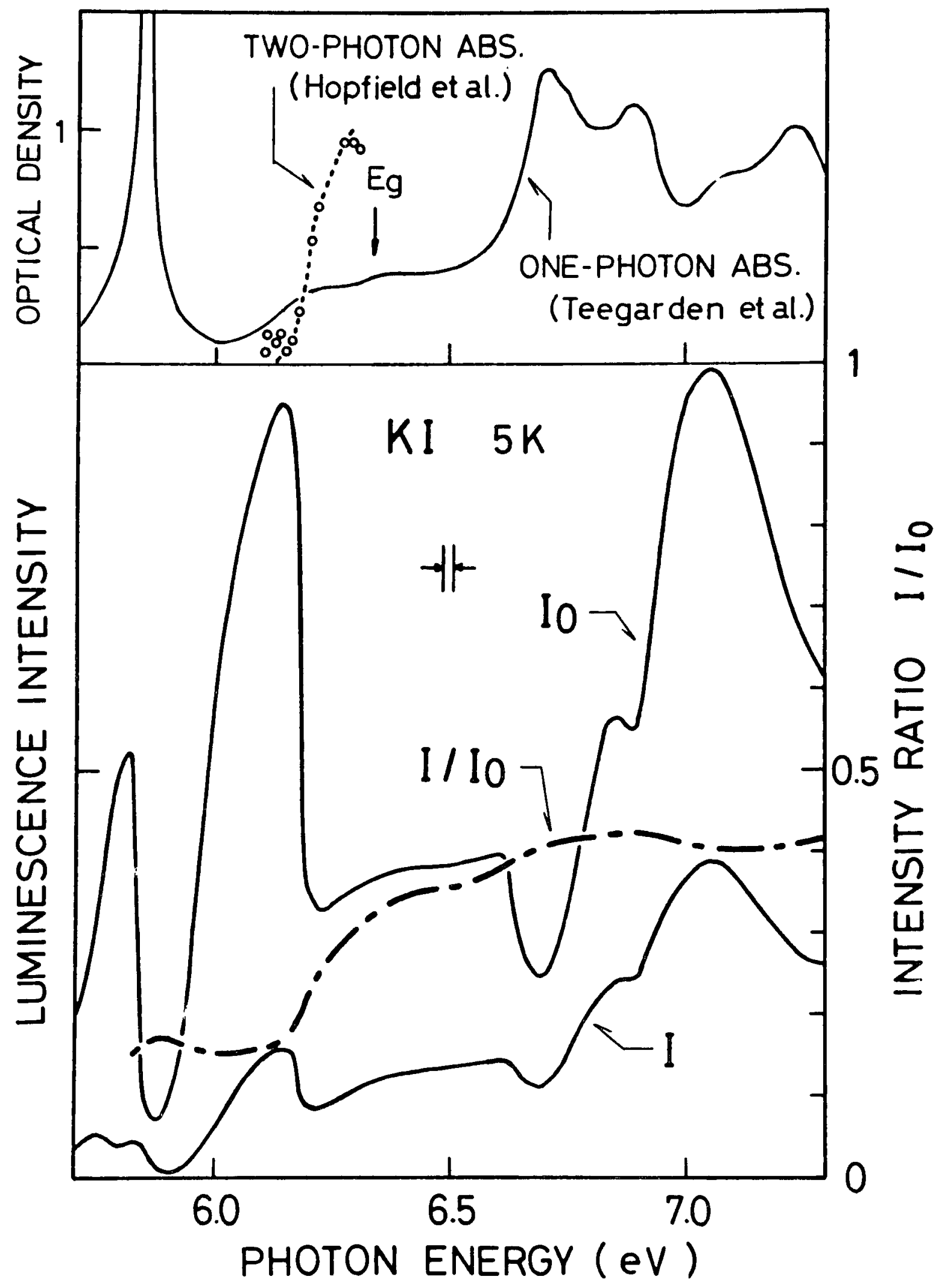

Fig. 1 


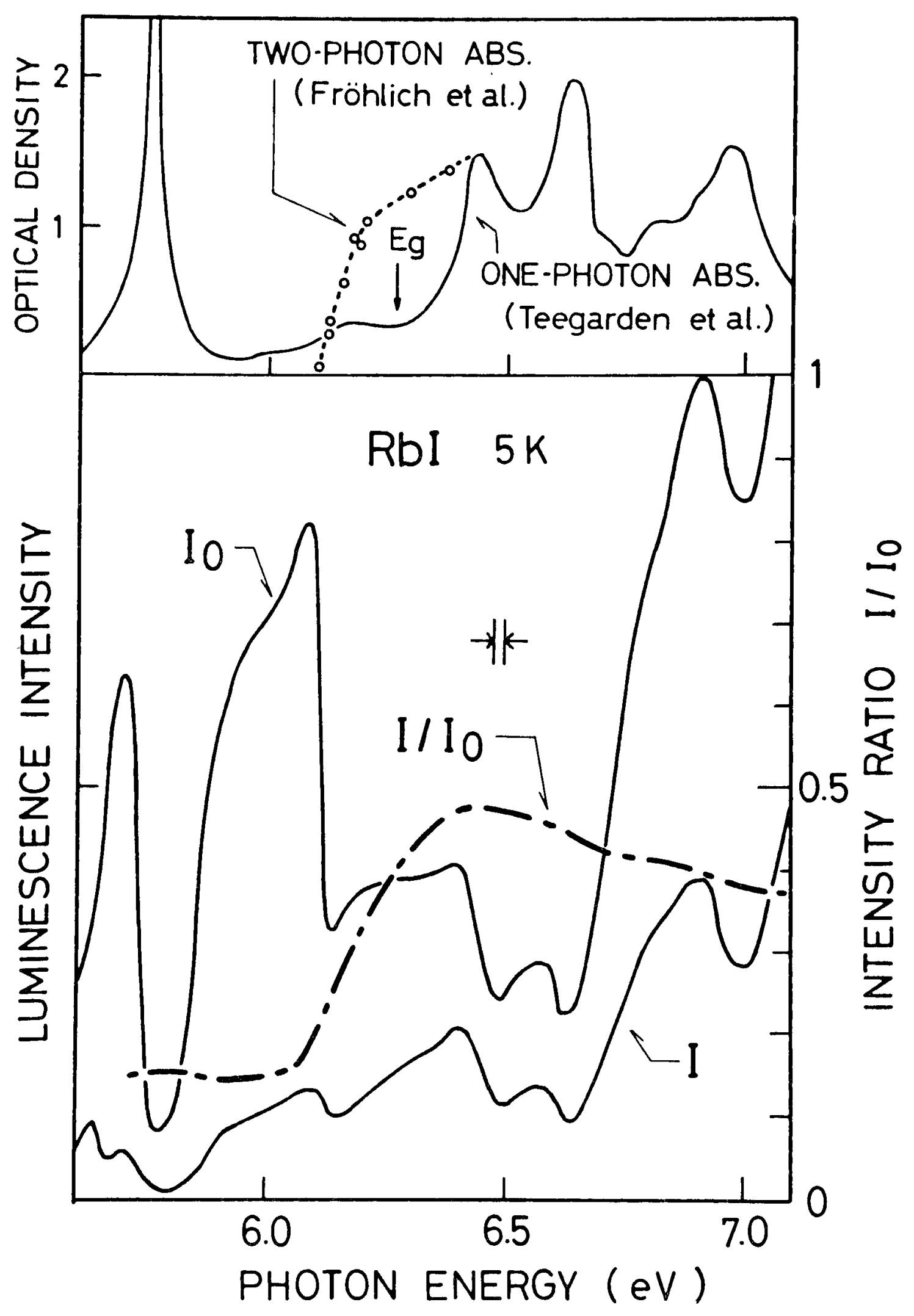

Fig. 2 


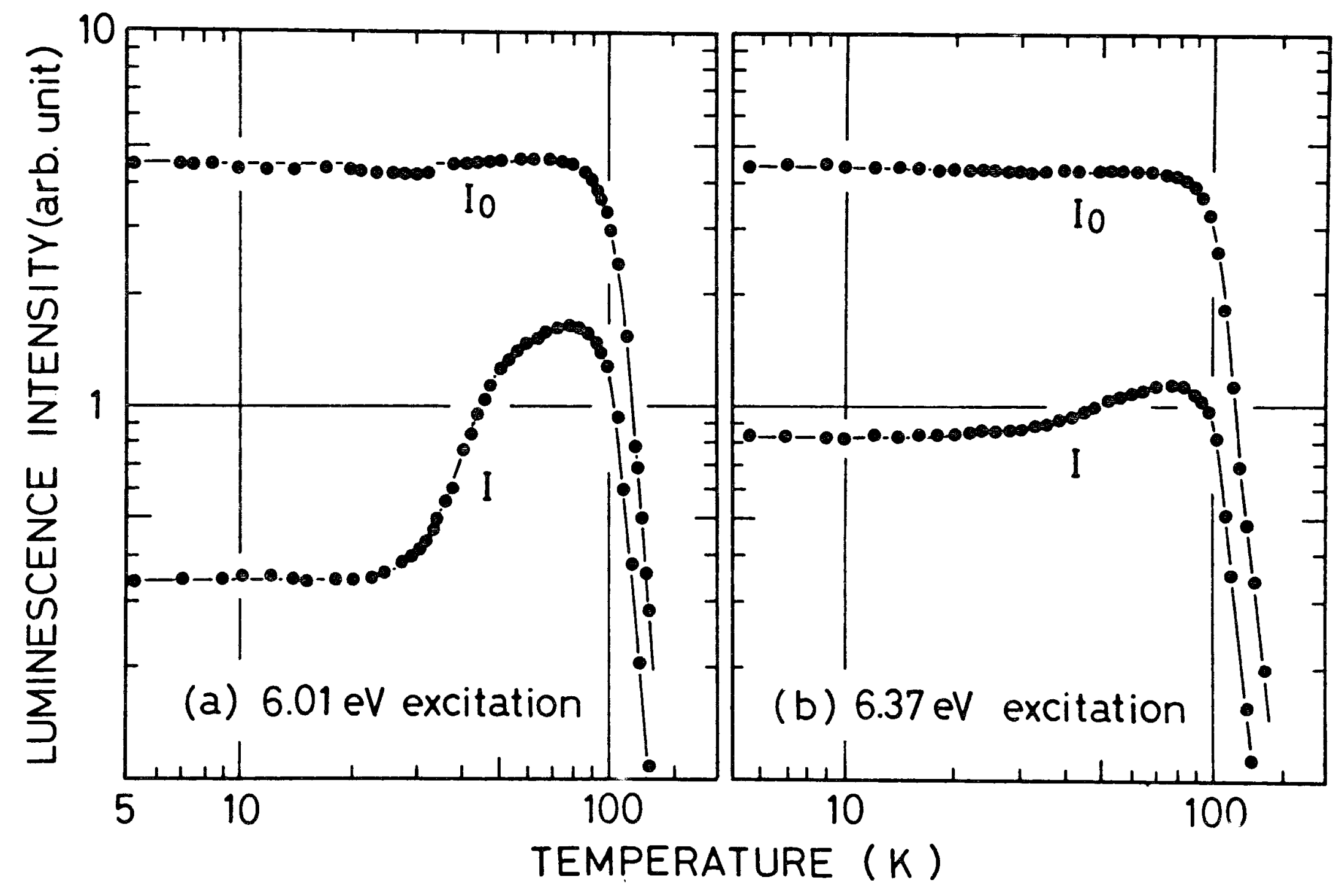

Fig. 3 


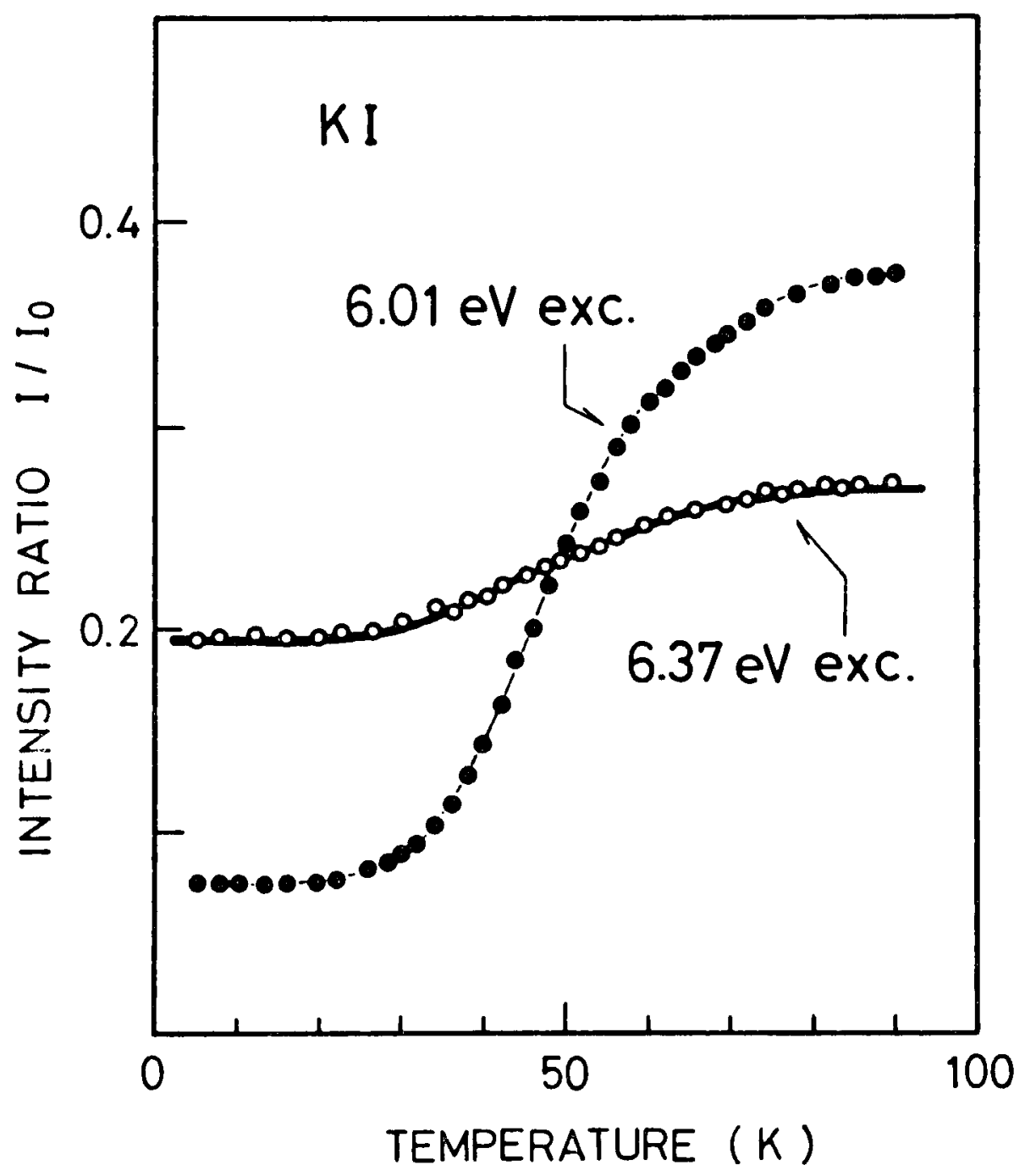

Fig. 4 


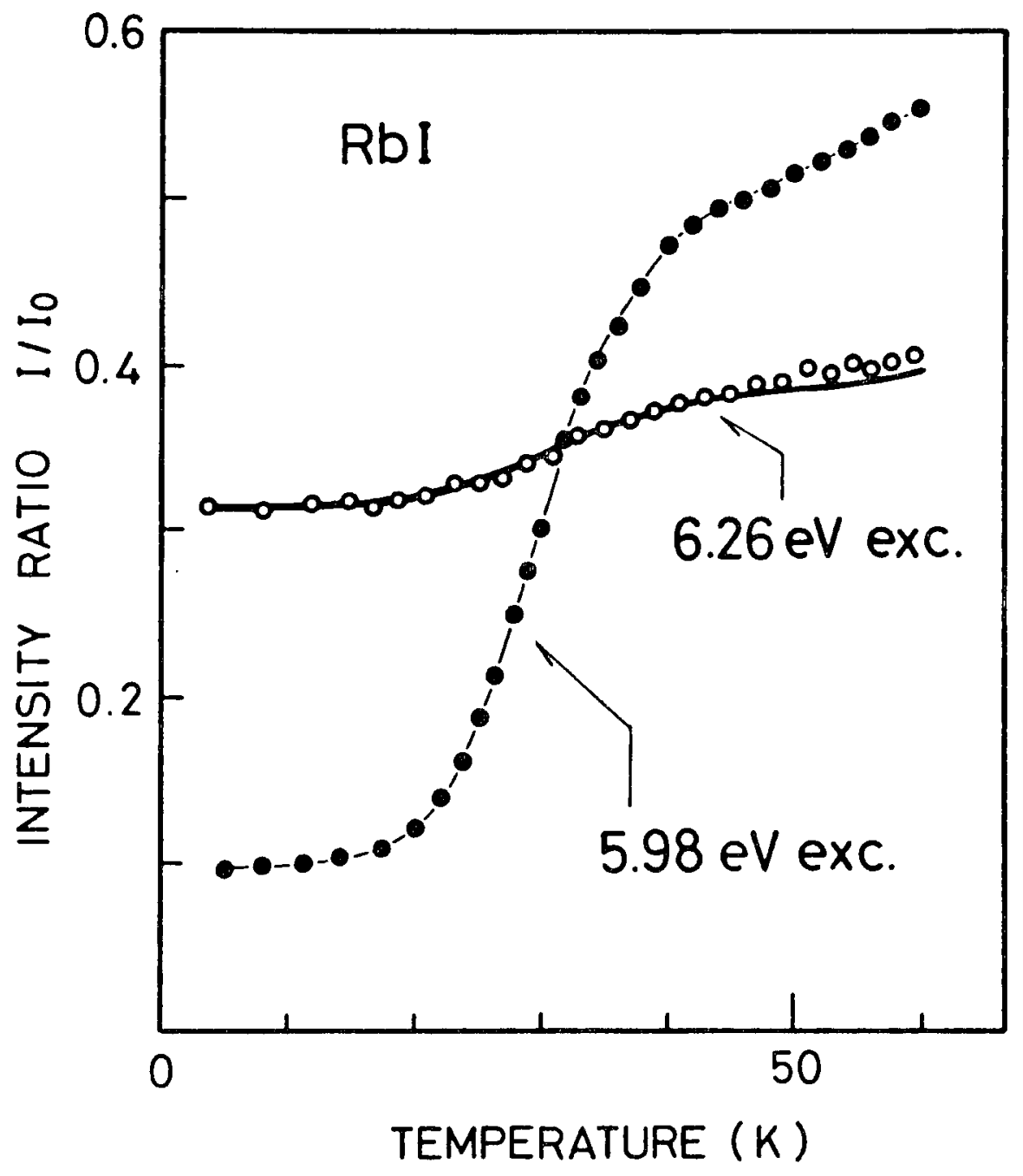

Fig. 5 


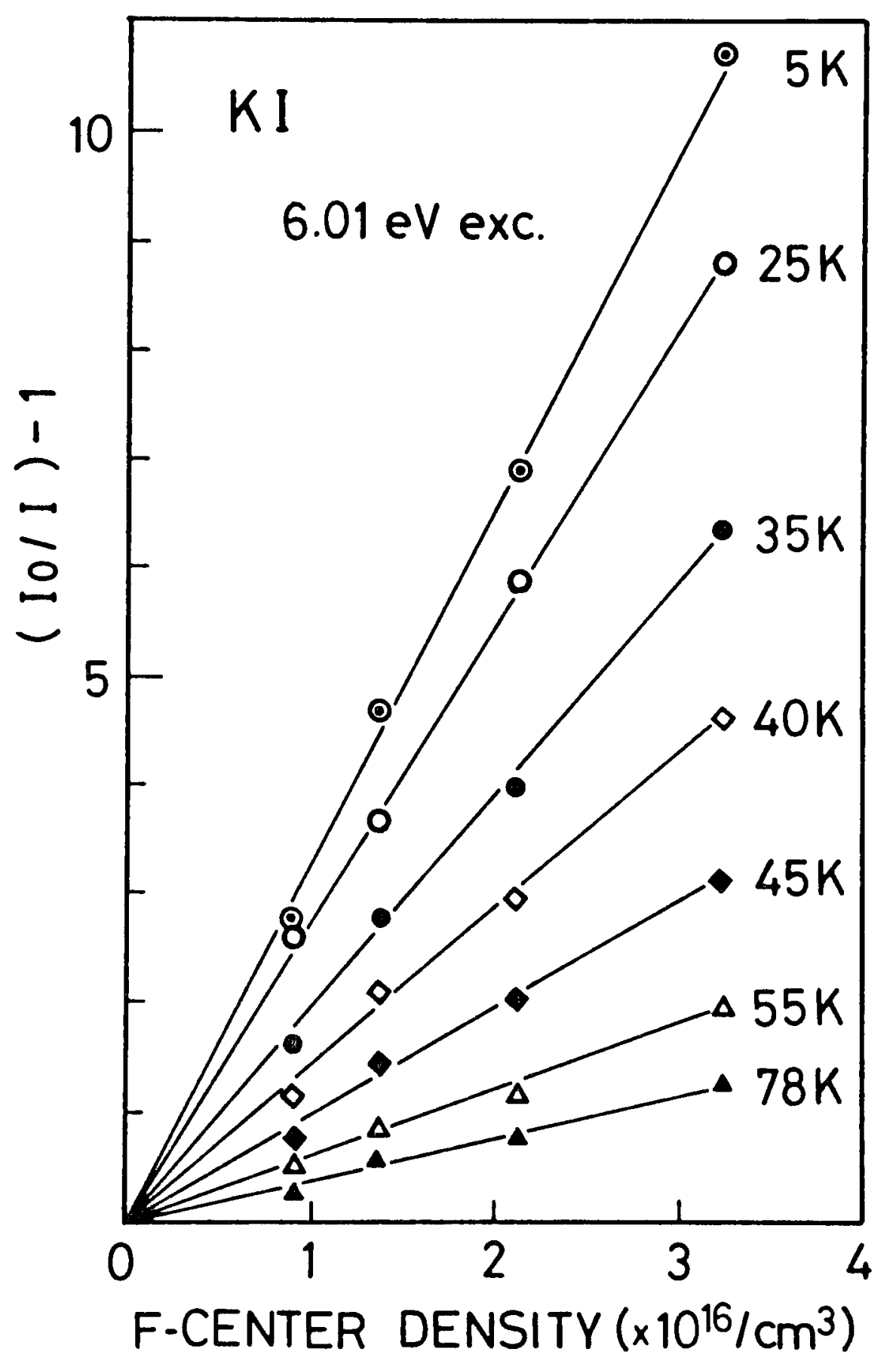

Fig. 6 


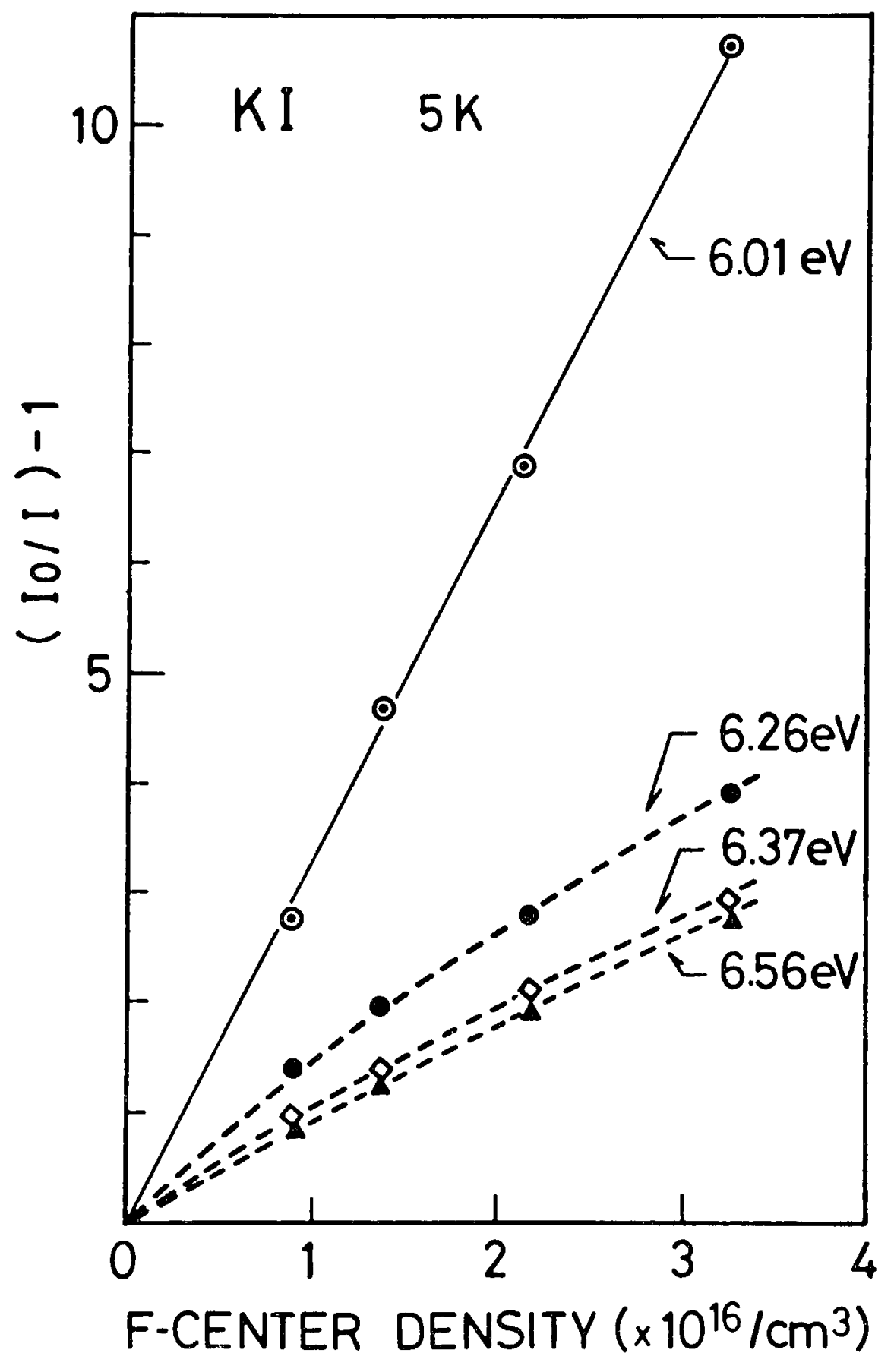

Fig. 7 


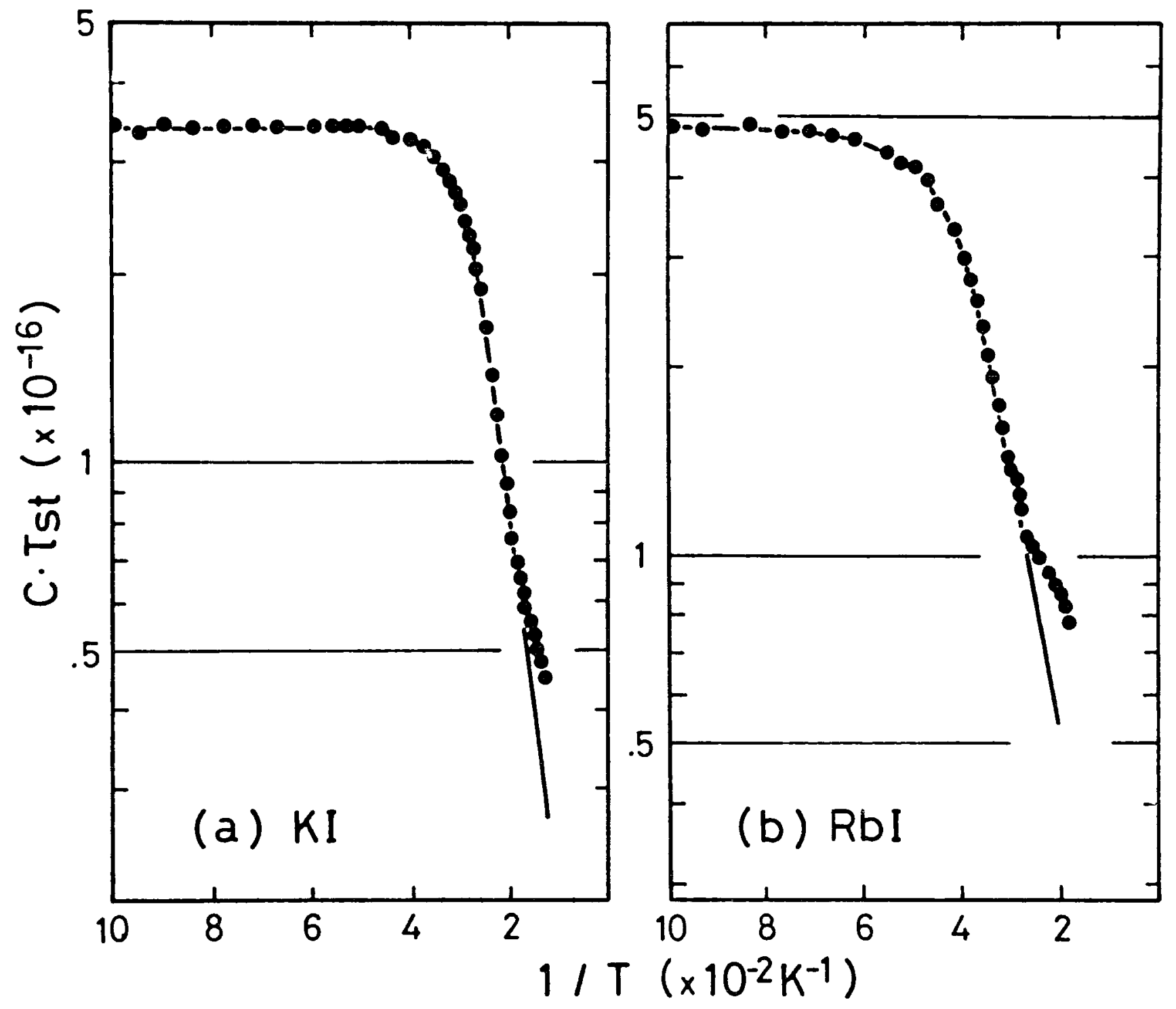

Fig. 8 